Original paper

\title{
Evaluation of optimization workflow using custom-made planning through predicted dose distribution for head and neck tumor treatment
}

\author{
Kentaro Miki ${ }^{a, *}$, Martijn Kusters ${ }^{\mathrm{b}}$, Takeo Nakashima ${ }^{\mathrm{c}}$, Akito Saito ${ }^{\mathrm{a}}$, Daisuke Kawahara ${ }^{\mathrm{d}}$, \\ Ikuno Nishibuchi $^{\text {a,d }}$, Tomoki Kimura ${ }^{\text {a }}$, Yuji Murakami ${ }^{\text {a,d }}$, Yasushi Nagata ${ }^{\text {a,d }}$ \\ a Department of Radiation Oncology, Hiroshima University Hospital, Hiroshima, Japan \\ ${ }^{\mathrm{b}}$ Department of Radiation Oncology, Radboud University Medical Center, Nijmegen, the Netherlands \\ ${ }^{\mathrm{c}}$ Radiation Therapy Section, Department of Clinical Support, Hiroshima University Hospital, Hiroshima, Japan \\ ${ }^{\mathrm{d}}$ Department of Radiation Oncology, Graduate School of Biomedical \& Health Sciences, Hiroshima University, Hiroshima, Japan
}

\section{A R T I C L E I N F O}

\section{Keywords:}

Radiotherapy

Treatment planning

Deep learning

Volumetric-modulated arc therapy

\begin{abstract}
A B S T R A C T
Purpose: Lack of a reference dose distribution is one of the challenges in the treatment planning used in volumetric modulated arc therapy because numerous manual processes result from variations in the location and size of a tumor in different cases. In this study, a predicted dose distribution was generated using two independent methods. Treatment planning using the predicted distribution was compared with the clinical value, and its efficacy was evaluated.

Methods: Computed tomography scans of 81 patients with oropharynx or hypopharynx tumors were acquired retrospectively. The predicted dose distributions were determined using a modified filtered back projection (mFBP) and a hierarchically densely connected U-net (HD-Unet). Optimization parameters were extracted from the predicted distribution, and the optimized dose distribution was obtained using a commercial treatment planning system.

Results: In the test data from ten patients, significant differences between the mFBP and clinical plan were observed for the maximum dose of the brain stem, spinal cord, and mean dose of the larynx. A significant difference between the dose distributions from the HD-Unet dose and the clinical plan was observed for the mean dose of the left parotid gland. In both cases, the equivalent coverage and flatness of the clinical plan were observed for the tumor target.

Conclusions: The predicted dose distribution was generated using two approaches. In the case of the mFBP approach, no prior learning, such as deep learning, is required; therefore, the accuracy and efficiency of treatment planning will be improved even for sites where sufficient training data are unavailable.
\end{abstract}

\section{Introduction}

In recent years, high-precision radiotherapy, such as intensitymodulated radiation therapy (IMRT) and volumetric modulated arc therapy (VMAT), has improved the dose concentration applied to tumors with complex shapes while reducing that added to normal tissue, compared with conventional three-dimensional radiotherapy $[1,2]$. The number of facilities for introducing such treatments has also increased. However, treatment planning and quality control are becoming more complicated with the advancements in irradiation methods. To improve efficiency and quality, automated treatment planning systems such as
Pinnacle $^{3}$ Auto-Planning (Philips Healthcare, Eindhoven, Netherlands) $[3,4]$ or Eclipse RapidPlan (Varian Medical Systems, Palo Alto, CA) [5,6] have been commercially released. However, the accuracy and safety of radiation treatment still depend on the planner's skill, particularly in complicated tumor sites, such as the head and neck, esophagus, or lung, because the optimization parameters used to achieve both an appropriate dose delivery to the planning target volume (PTV) and a reduced dose to the organ at risk (OAR) need to be set manually. However, this may cause quality differences between facilities. One of the difficulties in the optimization of treatment planning is the lack of a reference dose distribution. Because the tumor position and shape vary from patient to

\footnotetext{
* Corresponding author at: Department of Radiation Oncology, Hiroshima University Hospital, 1-2-3 Kasumi, Minami-ku, Hiroshima-shi, Hiroshima 734-8551, Japan.

E-mail address: kentaro-miki@hiroshima-u.ac.jp (K. Miki).
} 
patient, unless the optimization parameters are adjusted for each case, a treatment plan with sufficient accuracy cannot be obtained. As there is no goal dose distribution, optimization requires planner experience; therefore, accurate automation of treatment planning is difficult to achieve. If the ideal dose distribution is available, planners can simply optimize the distribution and adjust the dose constraints; thus, an accurate distribution can be easily obtained.

In a previous study, our group reported the development of a system that generates the ideal dose distribution by calculating only the target shape via application of the filtered back projection (FBP) method, which is traditionally used for computed tomography (CT) reconstruction [7]. In addition, a number of studies on a predicted dose distribution-generation system using deep learning techniques as a datadriven approach have been reported [8-10]. If these predicted dose distributions are used as reference, the parameters used for optimization can be extracted and adjusted automatically from the predicted distribution, and thus an efficient workflow for a custom-made treatment planning for each patient will be obtained.

The aim of this study was to confirm whether the quality of a treatment plan, generated by automatically extracting the optimization parameters through a predicted dose distribution, is equivalent to that of clinical use. In this study, the predicted dose distribution was generated using two independent methods, one based on an originally modified FBP (mFBP) method, and the other based on a deep learning method. These two methods have their own strengths and weaknesses. Confirming the differences in the characteristics of the predicted dose distribution generated by these two methods, treatment planning using the predicted dose distributions was compared with the clinical value, and its efficacy was evaluated. A schematic comparing our proposed planning workflow with a conventional approach is shown in Fig. 1.

\section{Materials and methods}

\subsection{Preparation of patient dataset}

We retrospectively studied 81 patients with oropharynx or hypopharynx tumors who were scheduled to receive VMAT radiation treatment at our facility from 2014 to 2017. Plan CT images were acquired using a 16-slice CT scanner (Light Speed ${ }^{\circledR}$ 16-slice; GE Healthcare, Waukesha WI, USA) and imported into the treatment planning system (TPS) (Eclipse, Varian Medical Systems, Palo Alto, CA). Scans were conducted using the tube voltage of $120 \mathrm{kV}$, tube current of $210 \mathrm{~mA}$, and slice thickness of $2.5 \mathrm{~mm}$. The tube current was modified or applied with auto-exposure control, if necessary. The PTV, brain, brain stem, mandible, oral cavity, left parotid, right parotid, spinal cord, and body shape were manually delineated by radiation oncologists. Prescribed doses of 70, 56, and 63 Gy were administered using a simultaneous integrated boost (SIB) technique to PTV70, which involved the primary tumor volume and lymph nodes with a certain margin for the risk region of the microscopic spread and a variation of the day-to-day patient setup; PTV56, which involved the regions at risk of a microscopic tumor; and PTV63, where the regions are judged as appropriate for a higher radiation dose than PTV56, respectively. The CT, delineated structures, and planning dose distribution used in clinical practice were exported in a Digital Imaging and Communications in Medicine (DICOM) format to the TPS. The Institutional Review Board of our hospital reviewed and approved this study.

\subsection{Dose distribution prediction using modified filtered back projection}

In a previous study, we developed and reported a predicted dose distribution generation method using an mFBP for a brain tumor [7]. Manually delineated target structures were acquired and placed within the 3-D voxel space. At this stage, 2-D calculations were conducted for each slice. Targets of PTV70, PTV63, and PTV56 were filled by their
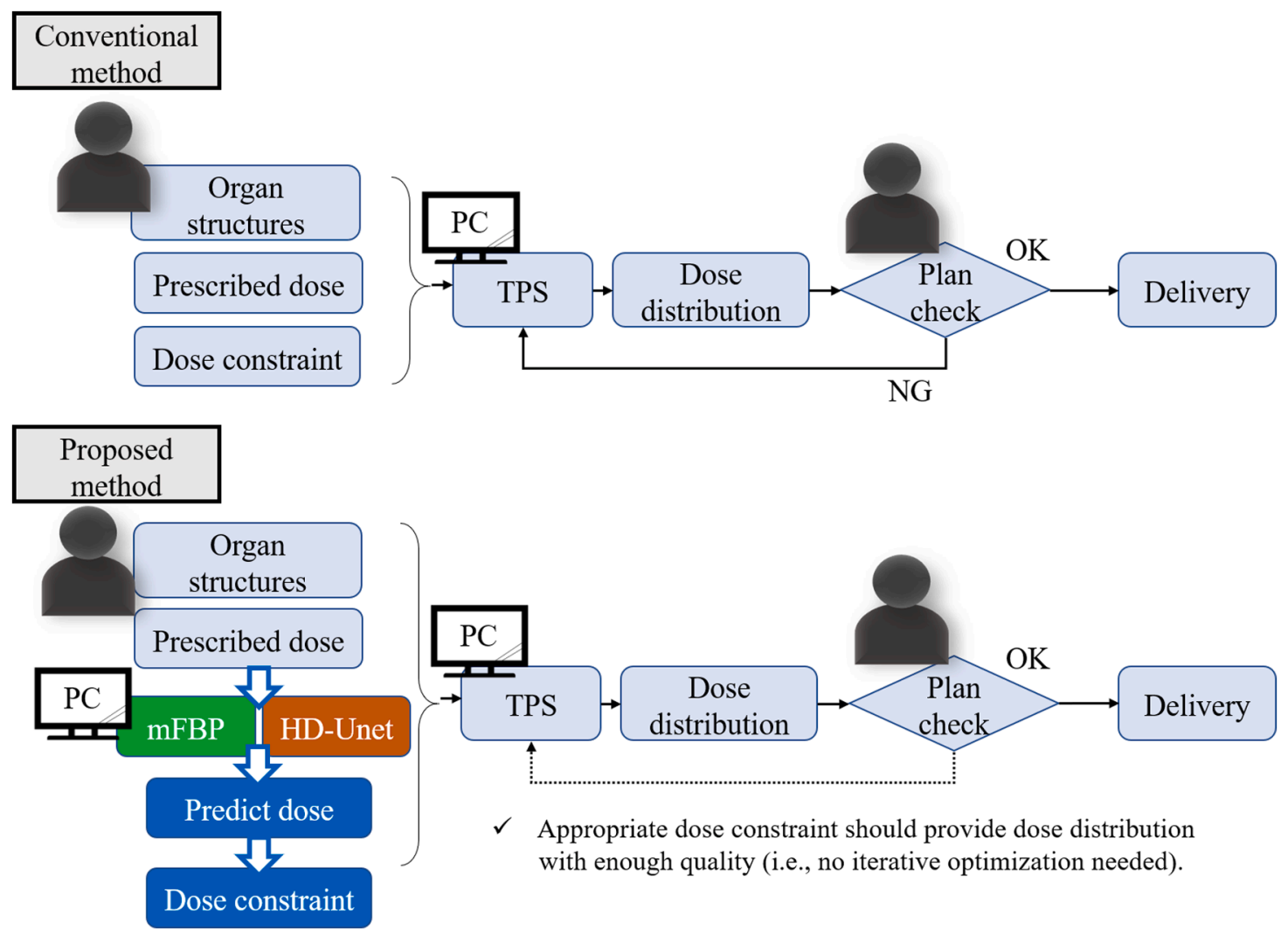

Fig. 1. Schematic of the workflow for predicted dose generation using modified filtered back projection (mFBP) method. 
prescribed dose values in the same 2-D plane $(f(x, y))$. A one-dimensional projection of PTVs along the X-ray path was calculated, and a Shepp-Logan filter for a back projection was applied. The same process was applied to the structures of the OARs, and the overlapping area was then subtracted from the 1-D projection of PTVs multiplied by the ratio $w$. The value of $w$ can be adjusted depending on the location of the tumor as a free parameter.

$g(\theta)=(1-w) \times g(\theta)_{P T V}, g(\theta)_{O A R} \neq 0$

where $\theta, g(\theta)_{P T V}$, and, $g(\theta)_{O A R}$ respectively express the gantry angle (i.e., beam direction), and inverse Fourier transforms of a 1-D projection of the PTV and the OAR, which are defined as follows:

$g(\theta)_{P T V, O A R}=\left(\int_{-\rho_{n}}^{\rho_{n}} P(\rho, \theta)_{P T V, O A R} H(\rho) e^{2 \pi i \rho s} d \rho\right)$,

where $P(\rho, \theta)_{P T V, O A R}, H(\rho), \rho_{n}$, and $s$ indicate the 1-D Fourier transform of a PTV or OAR projection, the filter function, the Nyquist frequency, and the distance of each pixel on a 2-D image from the origin $(s=x \cos \theta+$ $y \sin \theta$ ), respectively. As a result of several determinations, weight values $w$ of $0.99,0.99,0.60,0.50,0.10,0.10$, and 0.10 were used for the left parotid, right parotid, spinal cord, brain stem, oral cavity, mandible, and larynx, respectively. The inverse Fourier transform of a 1-D projection, $g(\theta)$, was multiplied by the tissue maximum ratio (TMR) to simulate the $\mathrm{X}$-ray attenuation inside the body. The distribution was calculated at every gantry angle $(d(x, y))$. See a previous study for more detailed formulas regarding mFBP [7]. Owing to the consideration of X-ray attenuation in the TMR and the removal of negative values from $g(\theta)$, the distribution superposed at multiple gantry angles does not contain uniform values within the PTV, unlike in CT reconstruction. In addition, as a limitation of our previous study [7], the approach used was not adaptable to SIB. To support the SIB as an update from our previous method, calculations are iteratively repeated until a sufficient uniformity is established within PTV70. The calculated distribution, $d(x, y)$, is normalized by the maximum voxel value of PTV70 for the prescribed dose.

$D(x, y)=d(x, y) \cdot \frac{\text { Prescribed dose (70Gy) }}{\text { maximum of PTV70 }}$

Subsequently, the difference in distribution from the prescribed dose is calculated as follows:

$f_{(2)}(x, y)=\left\{\begin{array}{cc}\left(D_{70 G y}+D_{63 G y}+D_{56 G y}\right)-a \cdot D(x, y) & , x, y \in P T V \\ 0 & , x, y \notin P T V\end{array}\right.$

where $a, D_{70 G y}, D_{63 G y}$, and $D_{56 G y}$ are represented as the scale factor and distributions at the prescribed doses of 70,63, and $56 \mathrm{~Gy}$, respectively (i. e., it should correspond to $f(x, y)$ ). The same process was repeated using $f_{(2)}(x, y)$ instead of $f(x, y)$. A schematic of this calculation is shown in Fig. 2 . The calculation was terminated when the number of iterations for each slice exceeded ten, or when three times the standard deviation in PTV70 was $2.5 \%$ or less. Finally, the calculated distribution was normalized to the dose at $95 \%$ of PTV70 (PTV70- $D_{95 \%}$ ), which is normally used in our hospital. The calculated distribution was converted into DICOM and imported into the TPS for use in the following treatment planning.

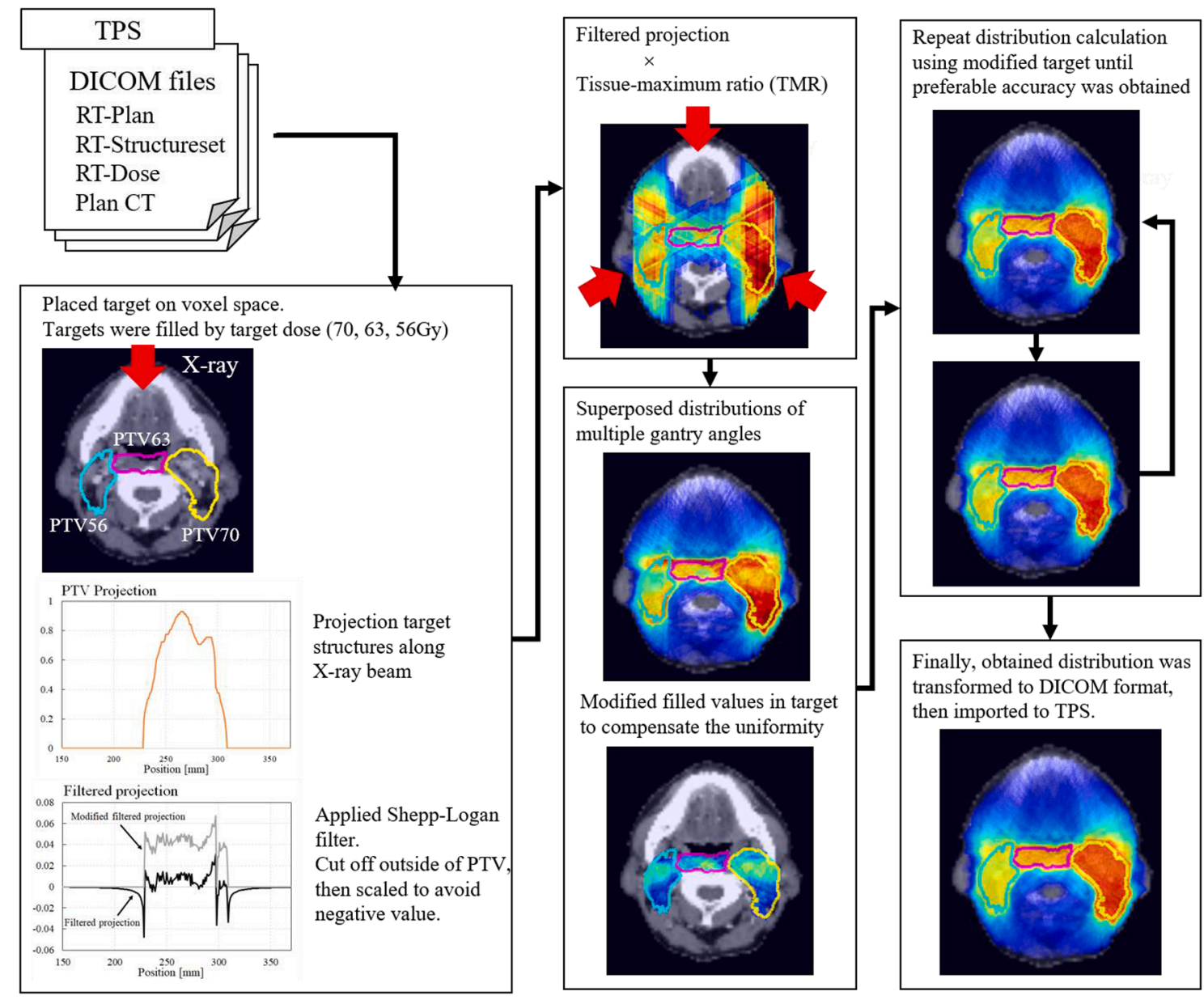

Fig. 2. Calculation workflow of mFBP method. 


\subsection{Predicted dose distribution using deep learning method}

To generate the predicted dose using a deep learning method, a hierarchically densely connected U-net (HD-Unet) architecture of a convolutional neural network was applied [9]. According to Nguyen et al., this model will produce a more accurate predicted dose distribution than a standard U-net [11]. This network is characterized by three operations: dense convolution, dense down-sample, and U-net up-sample. The dense convolution was concatenated for all previous feature maps after applying a normal convolution with a rectified linear unit (ReLU). The number of filters used for the convolution was set to 16 . Dense downsampling was applied through a concatenated $3 \times 3 \times 3$ convolution with a $2 \times 2 \times 2$ stride using a ReLU, and the down-sampled feature map with $2 \times 2 \times 2$ max pooling. U-net up-sampling was conducted through a concatenation of the feature map of a $2 \times 2 \times 2$ stride up-sample with a $3 \times 3 \times 3$ convolution to the same size feature map of the previous stage. The number of filters used for U-net up-sampling was 64 . Although there are some differences in the number of filters or pixels, these operations are similar to those proposed by Nguyen et al. Fig. 3 shows the series of architectures used in this study. The model was constructed using Python 3.6.0, tensorflow-gpu 1.11.0. As the workstation specifications, an Intel Core i7 (6 core) CPU, 32 GB of DDR4-2400 RAM, and a GeForce GTX 1080Ti 11 GB GPU were used.

The delineated structures of PTVs and OARs were resampled to a pixel resolution of $96 \times 96 \times 96$ and used as input. A clinical dose distribution was used for the teaching data, and the mean square error calculated for each pixel was used to determine the loss function as:

loss $=\frac{1}{n} \sum_{i=1}^{n}\left(y_{i, \text { predict }}-y_{i, \text { label }}\right)^{2}$.

The Adam optimizer [12] was applied, and the learning rate was set to 0.0001 . The number of batches to be learned simultaneously was set to 1 owing to the limitation of the GPU memory. A ReLU was used as the activation function. As a result of some determinations, regularization, batch normalization, and dropout were not used. From all datasets, ten cases were divided for testing, and 71 cases were used for training. The learning progress was confirmed using validation data that were placed in advance. A 7-fold cross-validation procedure was conducted for 71 cases of training data. The validation data were created by dividing the training data into ten datasets each, and the filter weights were initialized for each trial and replaced. The number of epochs was 300 for each trial. After training for 300 epochs, the accuracy of the model was evaluated using test data. Finally, the model with the highest test data accuracy was adopted, and the predicted dose distribution was generated using these ten test datasets. The patient data used for the test data are summarized in Table 1. The calculated distribution was converted into DICOM and imported into the TPS for use in the following treatment plan.

\subsection{Optimization of treatment planning using predicted dose distributions}

The treatment plan was optimized using the predicted dose distribution, which was generated using mFBP and HD-Unet. Using the same patient data as the HD-Unet test data, ten predicted doses using mFBP were also generated. As training data are not required in the mFBP study, 71 cases of training data in HD-Unet were not used in this study. The dose volume histogram (DVH) of each predicted dose was calculated, and the maximum (Dmax) and mean (Dmean) doses of the OARs were calculated and used as the parameters for re-optimization of the dose distribution using the TPS. In the predicted dose, regions surrounded by $20 \%-40 \%, 40 \%-60 \%$, and $60 \%-80 \%$ of the prescribed dose were extracted as pDose $20 \%$, pDose $40 \%$, and pDose $60 \%$, respectively, and used for optimization. Table 2 summarizes the specific optimization table used for each patient. The beam energy, collimator angle, arc angle range, and other field settings were commonly applied to the clinical plan, and an optimization was conducted only once using this patientspecific optimization table. The photon optimization (PO-13716, Varian Medical Systems, Palo Alto, CA) and Acuros XB (Varian Medical Systems, Palo Alto, CA) dose calculation algorithms were used. The calculated dose distribution was normalized to PTV70-D95\%. The homogeneity indexes (HIs) of PTV70, PTV63, and PTV56; Dmax of the brain stem and spinal cord; Dmean of the larynx, mandible, oral cavity, left parotid, and right parotid; and D2cc of the larynx, mandible, and oral cavity were calculated for the mFBP dose, HD-Unet dose, and clinical plan, respectively. The HI was defined using:

$\mathrm{HI}[\%]=\frac{D_{2 \%}-D_{98 \%}}{\text { Prescribed dose }} \times 100$

A statistical evaluation used for comparison was conducted using the Wilcoxon signed-rank test, which was $p<0.05$, considered statistically significant.

\section{Results}

\subsection{Predicted dose distribution using $m F B P$}

As an example, the predicted dose distribution generated using mFBP is shown in Fig. 4(a) and (d), (patient no. 3). The DVH of this calculated predicted dose and clinical plan is plotted in Fig. 5. PTV70-HI, PTV63HI, and PTV56-HI were $6.7 \%, 8.5 \%$, and $11.2 \%$ for the predicted dose using $\mathrm{mFBP}$; and $9.5 \%, 7.9 \%$, and $15.9 \%$, respectively, for the clinical

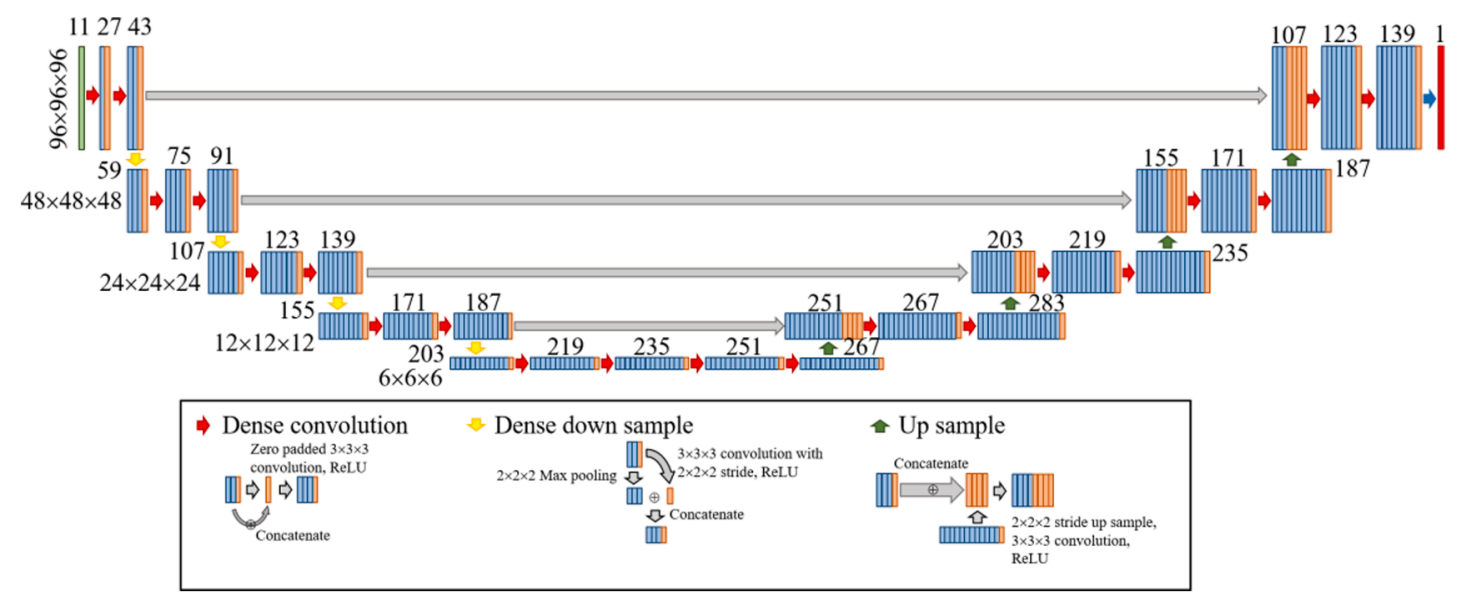

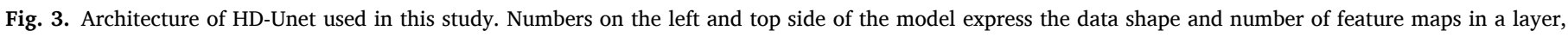
respectively. 
Table 1

Test data list for HD-Unet as deep learning approach, and for predicted dose generation using mFBP and HD-Unet.

\begin{tabular}{|c|c|c|c|c|c|c|c|c|}
\hline Patient ID & Tumor location & & $\mathrm{T}$ & $\mathrm{N}$ & M & Age & Male/Female & Mean square error \\
\hline 1 & Oropharynx & Left & 2 & $2 b$ & 0 & 65 & Male & 14.9 \\
\hline 2 & Oropharynx & Center & 2 & 0 & 0 & 67 & Male & 14 \\
\hline 3 & Hypopharynx & Left & $4 a$ & $2 \mathrm{~b}$ & 0 & 50 & Male & 9.6 \\
\hline 4 & Oropharynx & Right & 1 & $2 b$ & 0 & 58 & Female & 8.1 \\
\hline 5 & Hypopharynx & Left & $4 b$ & $2 b$ & 0 & 65 & Female & 6.8 \\
\hline 6 & Oropharynx & Left & 2 & $2 b$ & 0 & 55 & Male & 11.9 \\
\hline 7 & Oropharynx & Center & $4 a$ & 0 & 0 & 60 & Male & 8.1 \\
\hline 8 & Hypopharynx & Right & 2 & 3 & 0 & 56 & Male & 11.4 \\
\hline 9 & Oropharynx & Right & 2 & $2 \mathrm{a}$ & 0 & 78 & Female & 6.6 \\
\hline 10 & Hypopharynx & Center & $4 \mathrm{~b}$ & $2 c$ & 0 & 46 & Female & 9.4 \\
\hline Average & & & & & & 60 & & $10.1+/-2.9$ \\
\hline
\end{tabular}

Table 2

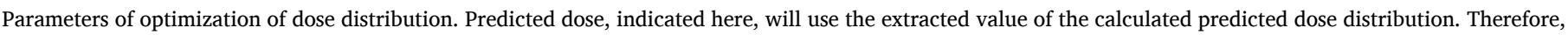

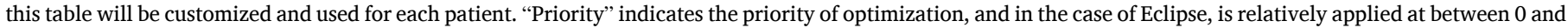
1,000 .

\begin{tabular}{|c|c|c|c|c|c|c|c|}
\hline \multirow[t]{2}{*}{ Target } & \multicolumn{2}{|c|}{ Constraint } & \multirow[t]{2}{*}{ Priority } & \multirow[t]{2}{*}{ Target } & \multicolumn{2}{|c|}{ Constraint } & \multirow[t]{2}{*}{ Priority } \\
\hline & & [cGy] & & & & [cGy] & \\
\hline \multirow[t]{2}{*}{ PTV70 } & Dmax & 7070 & 600 & Brain stem & Dmax & [predict dose] $\times 80 \%$ & 350 \\
\hline & Dmin & 7000 & 600 & Larynx & Dmax & [predict dose] $\times 100 \%$ & 150 \\
\hline \multirow[t]{2}{*}{ PTV63 } & Dmax & 6450 & 500 & & Dmean & [predict dose] $\times 90 \%$ & 200 \\
\hline & Dmin & 6300 & 500 & Mandible & Dmax & [predict dose] $\times 100 \%$ & 150 \\
\hline \multirow[t]{2}{*}{ PTV56 } & Dmax & 5750 & 500 & & Dmean & [predict dose] $\times 90 \%$ & 200 \\
\hline & Dmin & 5600 & 500 & Oral cavity & Dmax & [predict dose] $\times 100 \%$ & 150 \\
\hline pDose $60 \%$ & Dmax & 5600 & 200 & & Dmean & [predict dose] $\times 90 \%$ & 200 \\
\hline pDose $40 \%$ & Dmax & 4200 & 200 & Left parotid & Dmax & [predict dose] $\times 100 \%$ & 150 \\
\hline \multirow[t]{4}{*}{ pDose $20 \%$} & Dmax & 2800 & 200 & & Dmean & [predict dose] $\times 70 \%$ & 250 \\
\hline & & & & Right parotid & Dmax & [predict dose] $\times 100 \%$ & 150 \\
\hline & & & & & Dmean & [predict dose] $\times 70 \%$ & 250 \\
\hline & & & & Spinal code & Dmax & [predict dose] $\times 80 \%$ & 350 \\
\hline
\end{tabular}

Abbreviations: Dmax = maximum dose Dmin $=$ minimum dose; Dmean $=$ mean dose.

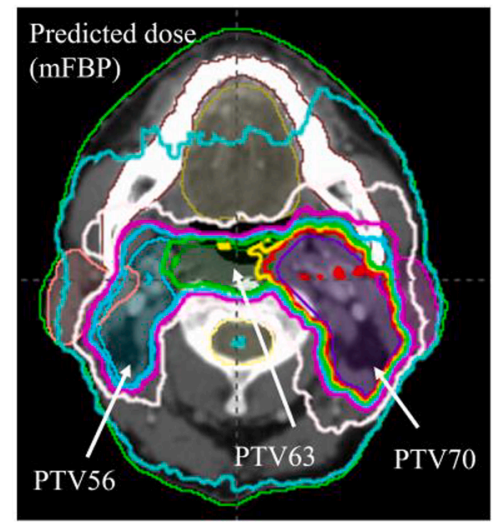

(a)

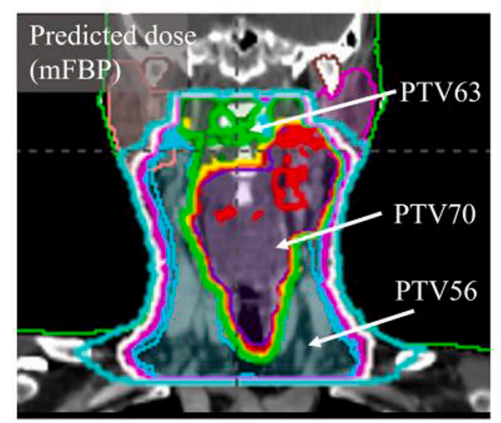

(d)

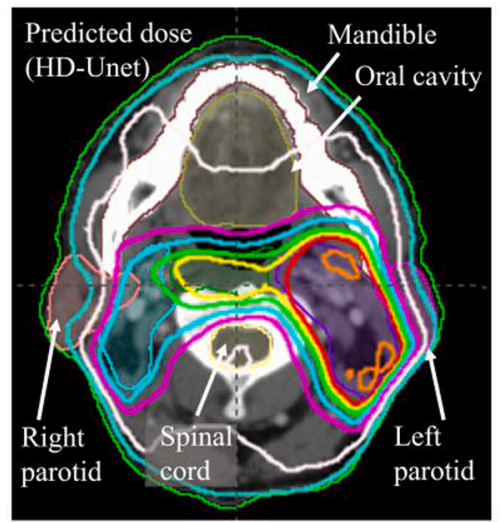

(b)

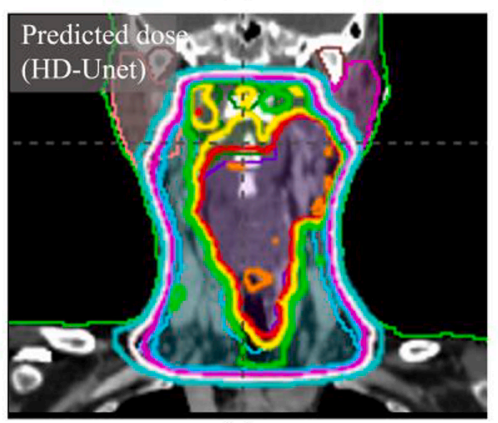

(e)

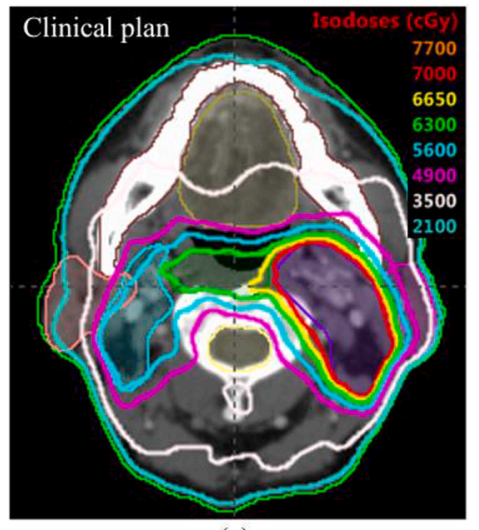

(c)

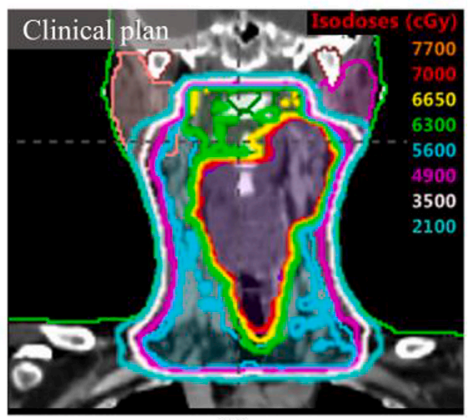

(f)

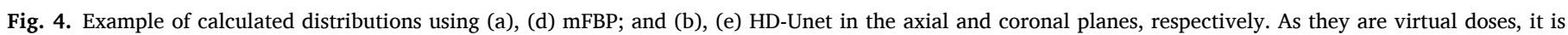
impossible to irradiate these distributions. (c), (f) Clinical plan for reference (patient no. 3). 


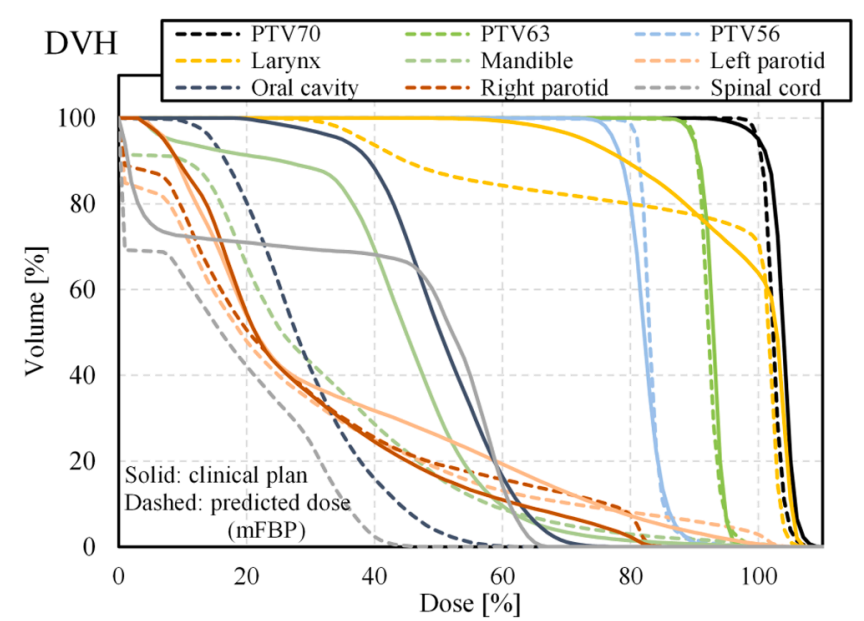

Fig. 5. Dose volume histogram (DVH) of predicted doses using mFBP and clinical plan.

plan. The Dmean of the larynx, mandible, oral cavity, left parotid, and right parotid were 63.5, 21.6, 20.4, 19.7, and $20.2 \mathrm{~Gy}$ for the predicted dose, and $68.0,30.8,35.3,23.2$, and 20.4 Gy for the clinical plan, respectively. The Dmax of the spinal cord was 35.6 and $47.8 \mathrm{~Gy}$ for the predicted dose and clinical plan, respectively.

\subsection{Predicted dose distribution using HD-Unet}

The mean square error as the loss function for deep learning of the pixel values between the output of HD-Unet and the teaching images is plotted in Fig. 6 as a function of the number of epochs. The bandwidth in Fig. 6 represents the maximum and minimum values in the 7-hold crossvalidation trials, and the line represents the average value. As an example, Fig. 4 (b) and (e) show the predicted dose distribution, generated by HD-Unet, in the axial and coronal planes, respectively. In addition, the dose distributions of the clinical plan are shown in Fig. 4 (c) and (f) for reference.

\subsection{Optimization of treatment planning using predicted dose distribution}

The re-optimized dose distribution using commercial TPS utilizing the predicted dose of mFBP (mFBP dose) and HD-Unet (HD-Unet dose) is shown in Fig. 7 (a) and (b). Fig. 7 (c) shows the clinical plan as a reference. The DVHs of the MFBP and HD-Unet doses are shown in Fig. 7 (d) and (e) along with a clinical plan. PTV70-HI, PTV63-HI, and PTV56HI were $8.6 \%, 9.6 \%$, and $12.5 \%$ for the $\mathrm{mFBP}$ dose; $6.5 \%, 7.4 \%$, and

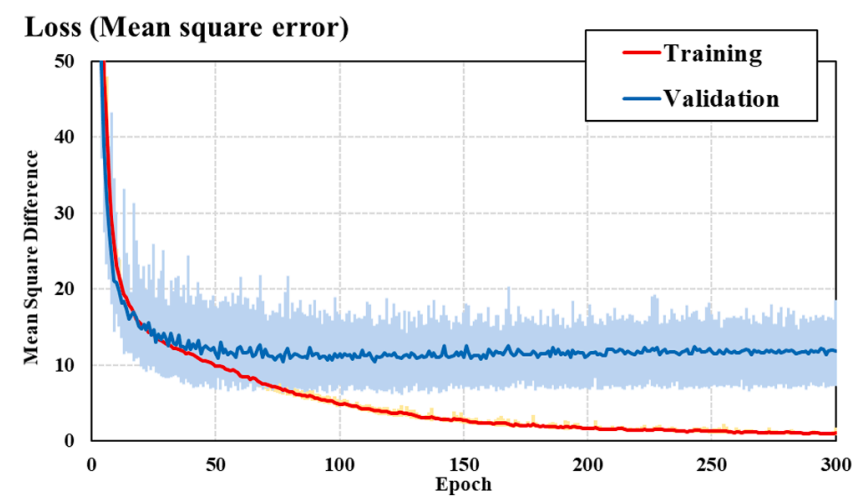

Fig. 6. Mean square error as the loss function for deep learning of pixel values between output of HD-Unet and teaching images. The bandwidth represents the maximum and minimum values in 7-hold cross-validation trials and tests, and the line represents the average value.
9.8\% for the HD-Unet dose, and 9.5\%, 7.9\%, and $15.9 \%$ for the clinical plan, respectively. The Dmean of the larynx, mandible, oral cavity, left parotid, and right parotid were 67.1, 31.0, 30.7, 19.9, and $18.9 \mathrm{~Gy}$ for the mFBP dose; 65.9, 32.7, 36.6, 19.5, and 16.4 Gy for the HD-Unet dose; and $68.0,30.8,35.3,23.2$, and $20.4 \mathrm{~Gy}$ for the clinical plan, respectively. Moreover, the Dmax of the spinal cord was $36.8,47.5$, and 47.8 Gy for the mFBP dose, HD-Unet dose, and clinical plan, respectively.

The average dose assessments of ten patients are summarized in Fig. 8 (a). The bar on the data represents the standard deviation. Differences between the predicted dose method and clinical plan with a statistical significance are shown in Fig. 8 (a) along with the p-value. The average and standard deviation of the difference between the predicted dose plan and the clinical plan are shown in Fig. 8 (b).

\section{Discussion}

In this study, we developed a new optimization workflow by applying the predicted dose distributions using mFBP and HD-Unet for VMAT planning. As these predicted doses have a virtual distribution, they cannot be used directly in clinical practice. However, by extracting the optimization parameters from these distributions, they can be used for calculations in a usual TPS. The results shown in Fig. 8 indicate that the predicted dose plan exhibits the same or superior plan quality compared with the clinical plan. As the predicted dose plan does not require trial and error (only one routine optimization calculation is required), an effective workflow plan will be established for VMAT with sufficient quality compared to a conventional method, which is generated after an extensive consideration by experts over a sufficient amount of time. In this study, DVH and its values were mainly used to evaluate the dose distribution quality, as shown in Figs. 7 and 8. Although gamma analysis is widely used to compare the matches of distributions, the aim of this study is to produce not only an equivalent distribution, but also a better distribution than that from a clinical plan. Thus, comparison using DVH for evaluation of the quality of dose distribution would be better than using gamma analysis, which only indicates the match of distributions. We confirmed that our strategy can be implemented in clinical practice for head and neck tumor cases as a feasible tool to efficiently determine treatment quality.

To confirm that the mFBP method can obtain the same or better results than deep learning, we developed a unique method for generating the predicted dose and compared it with a method using a deep learning model, which has become popular in recent years. From Fig. 4, a characteristic difference between the predicted doses generated by the two methods can be observed: the dose generated by mFBP indicates an extremely tight dose distribution for PTV but offers some unrealistic goals for the OARs. In contrast, although it seems that deep learning reproduces the clinical plan relatively better than $\mathrm{mFBP}$, the coverage for the PTV is slightly insufficient, which is caused by saturation of the validation or test data (i.e., an overfitting) at a certain value, as shown in Fig. 6. Deep learning requires a large amount of data to build an accurate model without overfitting; therefore, the result significantly depends on the amount and quality of the input data. Although increasing data size may improve overfitting, there is a limit to collecting large amounts of clinical data. Prior to this study, we compared HD-Unet with a standard U-net for adoption in our research but did not find any differences in their accuracies, as reported by Nguyen et al. [9]. This may also be due to the number of data on the patient cases or structures used. As deep learning is an evolving technology, a method may be improved in terms of accuracy by developing a better algorithm or architecture. However, re-learning is necessary each time the dataset used for input differs even trivially, such as the number of pixels, the type of organ structure, or the tumor location. As the training data, oropharyngeal and hypopharyngeal data were used together in this study, although it might have been better to use them separately for greater accuracy. Therefore, to employ this approach in several facilities, it is necessary to prepare a dataset according to common rules in advance, which is one of the 


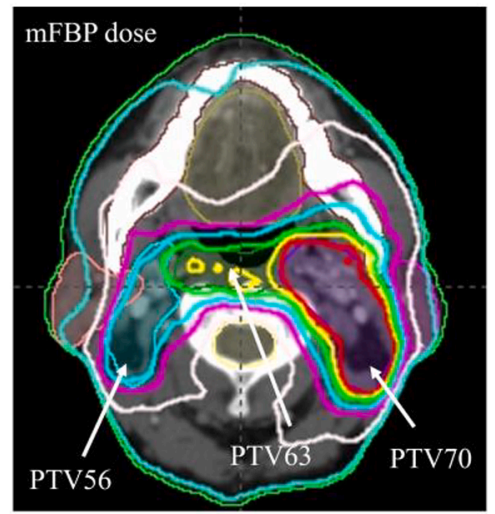

(a)

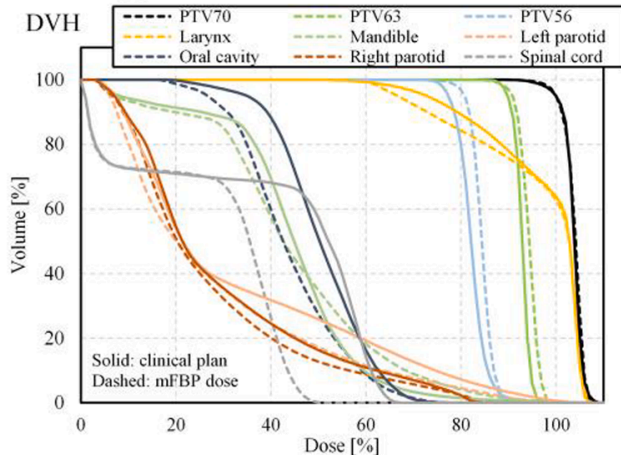

(d)

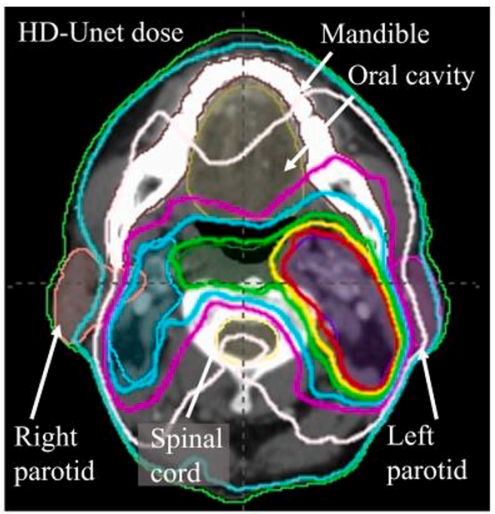

(b)

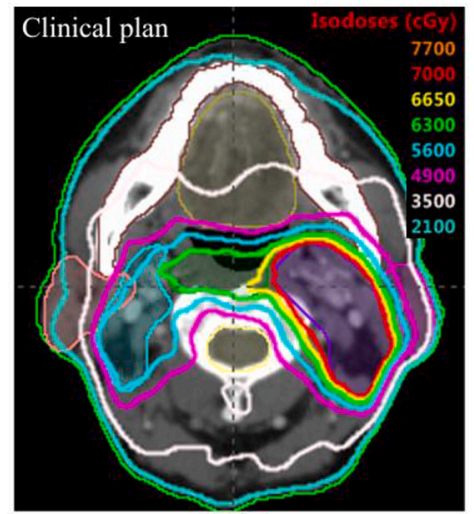

(c)

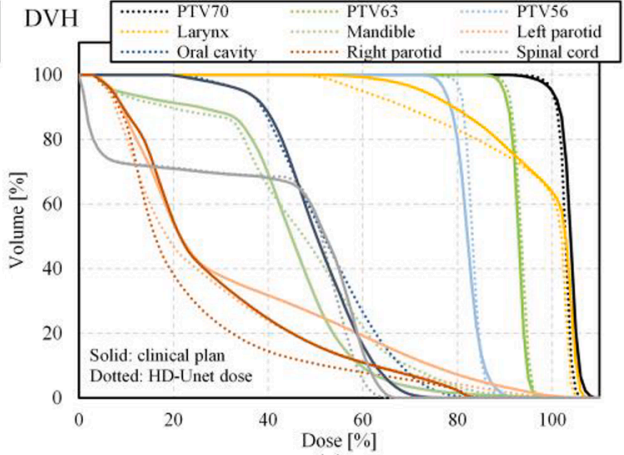

(e)

Fig. 7. Dose distribution, which can be re-optimized using custom-made parameter table (i.e., Table 2), for specific patient using (a) mFBP and (b) HD-Unet (patient no. 3). (c) Clinical plan for reference. DVHs of the mFBP and HD-Unet doses compared with the clinical plan are shown in (d) and (e).

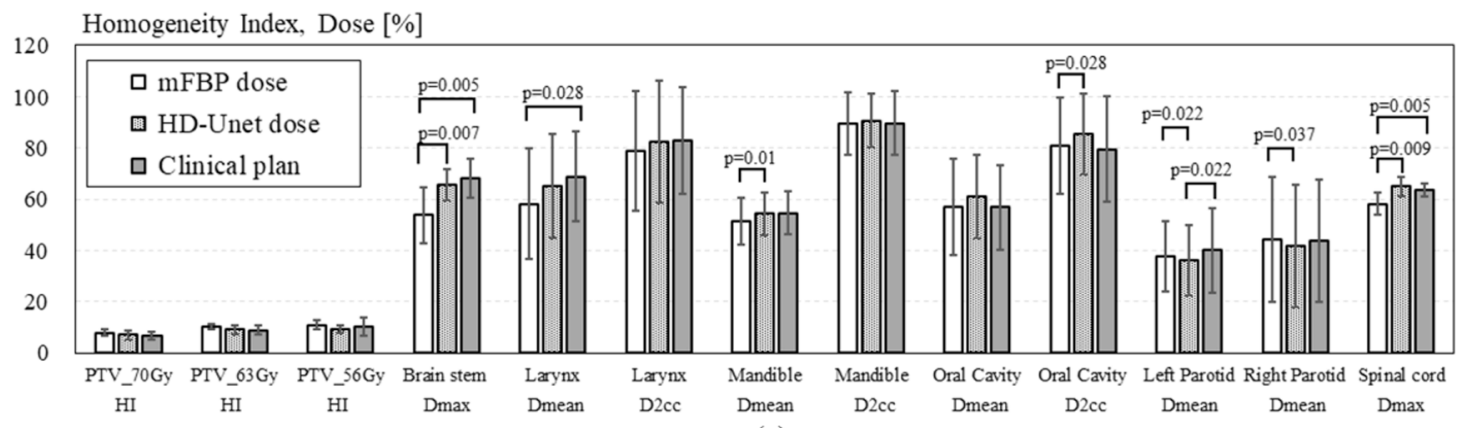

(a)

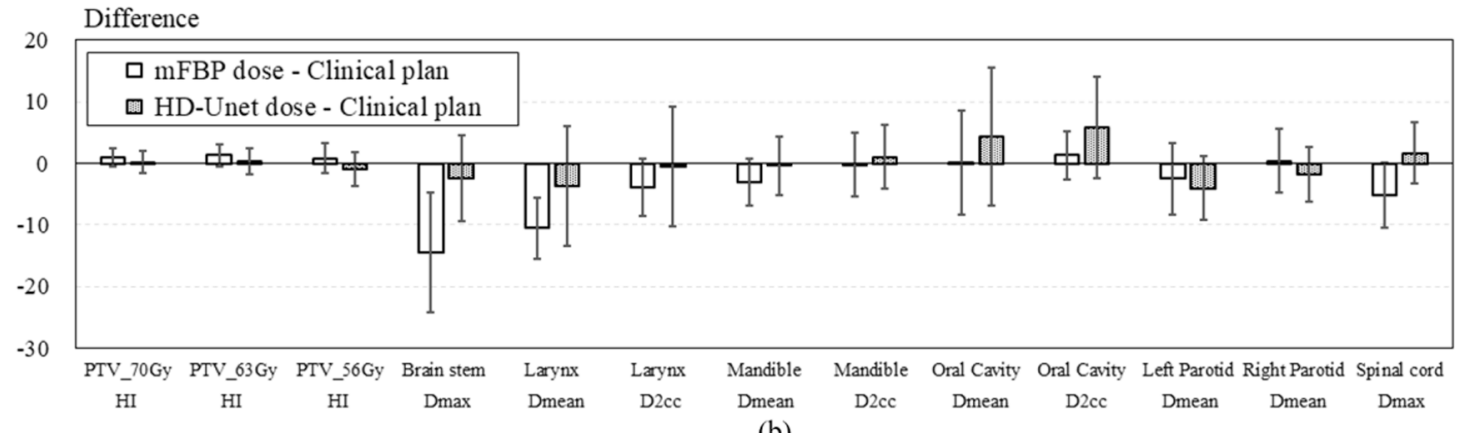

(b)

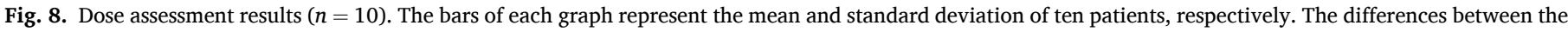
mFBP or HD-Unet dose in the clinical plan were calculated for each patient, and the mean and standard deviation are shown in (b).

disadvantages of current deep learning approaches. With our proposed mFBP, it is not necessary to use training data for pre-learning, and thus it is easy to expand to other facilities. An advantage of $\mathrm{mFBP}$ is that it does not require a large amount of training data and can generate a dose distribution with a constant quality without relying on past clinical data.

Similar to the purpose of this study, Plan IQ (Sun Nuclear Corp., Melbourne, FL, USA), which is an efficient method used in treatment planning, has recently been commercialized and evaluated $[13,14]$. This 
method first creates an ideal dose distribution for the PTV, called a benchmark 3D dose, considering the penumbra of the treatment beam, attenuation, and scatter related to the location relationship among the PTV, OARs, and other organs. The DVH of the 3D dose distribution is calculated and used for optimization of the treatment planning (which herein is called the feasibility DVH and corresponds to Fig. 5 regarding our method). The concept of generating an ideal dose distribution and promoting optimization by providing an achievable DVH is common to our method; however, as described, the calculation method differs, i.e., application of the mFBP method to the ideal fluence distribution is a feature of our approach. The advantage of this method over Plan IQ is that it can be used for treatment planning when applying an asymmetric beam angle, such as a half-arc or non-coplanar irradiation because it simulates X-ray irradiation from outside the body. In addition, mFBP can provide a virtual dose distribution as well as a feasible DVH; thus, it can utilize the shape of the distribution for optimization, such as pDose $20 \%$ described in this study. Our algorithm can now be applied to SIBs; therefore, it can be expected that it will be effective for not only head and neck, but also sites that often use an asymmetric beam angle such as lung, esophageal, or brain tumors.

Studies on efficient VMAT treatment planning, which are based on automated planning processes using commercially available software such as Pinnacle ${ }^{3}$ Auto-Planning (Philips Healthcare, Eindhoven, Netherlands) $[3,4]$ and RapidPlan (Varian Medical Systems, Palo Alto, CA) $[5,6]$, examinations regarding the tradeoff between PTV and OARs through multicriteria optimization [15-17], or Plan IQ, as described previously, could be used efficiently with our proposed method. This is because, by providing an ideal dose distribution, the method introduced in this study can be used as an input for such software. To introduce the approach using the predicted dose distribution, and establish an efficient and safe treatment plan for other clinical sites, it is necessary to conduct a continuous study on the effective use of such current products.

Because a deep learning method as a data-driven approach and the mFBP method as a theoretical approach have their own advantages and disadvantages, multifaceted research for generating the predictive dose will be effective. By fusing these different approaches, it may be possible to generate a high-quality predicted dose while using a small amount of clinical data in the future.

\section{Conclusion}

We confirmed that a predicted dose distribution can be generated through two independent approaches by applying FBP and deep learning from only a structural dataset. A level of quality equivalent to that of clinical planning can be established using only one optimization calculation. As a feature of the mFBP approach, no prior learning is required, such as deep learning, which will improve the accuracy and efficiency of treatment planning even for sites where sufficient training data are unavailable.

\section{Note}

This work was supported by JSPS KAKENHI Grant Number 20K08079.

\section{Declaration of Competing Interest}

The authors declare that they have no known competing financial interests or personal relationships that could have appeared to influence the work reported in this paper.

\section{References}

[1] Otto K. Volumetric modulated arc therapy: IMRT in a single gantry arc: Single arc radiation therapy. Med Phys 2008;35(1):310-7. https://doi.org/10.1118/ 1.2818738 .

[2] Verbakel WFAR, Cuijpers JP, Hoffmans D, Bieker M, Slotman BJ, Senan S. Volumetric Intensity-Modulated Arc Therapy Vs. Conventional IMRT in Head-andNeck Cancer: A Comparative Planning and Dosimetric Study. Int J Radiat Oncol Biol Phys 2009;74(1):252-9. https://doi.org/10.1016/j.ijrobp.2008.12.033.

[3] Kusters J, Bzdusek K, Kumar P, et al. Automated IMRT planning in Pinnacle-A study in head-and-neck cancer. Strahlenther Onkol 2017;193(12):1077-8.

[4] McConnell KA, Marston T, Zehren BE, et al. Dosimetric evaluation of Pinnacle's automated treatment planning software to manually planned treatments. Technol Cancer Res T 2018;17:1-7.

[5] Kubo K, Monzen H, Ishii K, Tamura M, Kawamorita R, Sumida I, Mizuno H, Nishimura Y. Dosimetric comparison of RapidPlan and manually optimized plans in volumetric modulated arc therapy for prostate cancer. Physica Med 2017;44: 199-204. https://doi.org/10.1016/j.ejmp.2017.06.026.

[6] Fogliata A, Cozzi L, Reggiori G, Stravato A, Lobefalo F, Franzese C, Franceschini D, Tomatis S, Scorsetti M. RapidPlan knowledge based planning: iterative learning process and model ability to steer planning strategies. Radiat Oncol 2019;14(1). https://doi.org/10.1186/s13014-019-1403-0.

[7] Miki K, Hioki K, Nakashima T, Saito A, Murakami Y, Kimura T, Nishibuchi I, Nagata Y. Development of geometrically ideal dose distribution as a reference for treatment planning in VMAT using filtered back-projection method. Physica Med 2019;57:115-22. https://doi.org/10.1016/j.ejmp.2018.12.034.

[8] Kearney V, Chan JW, Haaf S, Descovich M, Solberg TD. DoseNet: A volumetric dose prediction algorithm using 3D fully-convolutional neural networks. Phys Med Biol 2018;63(23):235022.

[9] Nguyen D, Jia X, Sher D, et al. 3D radiotherapy dose prediction on head and neck cancer patients with a hierarchically densely connected U-net deep learning architecture. Phys Med Biol 2019;64(6):065020.

[10] Nguyen D, Long T, Jia X, Lu W, Gu X, Iqbal Z, Jiang S. A feasibility study for predicting optimal radiation therapy dose distributions of prostate cancer patients from patient anatomy using deep learning. Sci Rep 2019;9(1). https://doi.org/ 10.1038/s41598-018-37741-x.

[11] Olaf R, Philipp F, Thomax B. U-Net: Convolutional networks for biomedical image segmentation. International Conference on Medical Image Computing and Computer-Assisted Intervention. 2015.234-241.

[12] Kingma D, Ba J. Adam: A method for stochastic optimization. International Conference on Learning Representations; 2014.

[13] Ahmed S, Nelms B, Gintz D, Caudell J, Zhang G, Moros EG, Feygelman V. A method for a priori estimation of best feasible DVH for organs-at-risk: Validation for head and neck VMAT planning. Med Phys 2017;44(10):5486-97. https://doi.org/ 10.1002/mp.12500.

[14] Perumal B, Sundaresan HE, Ranganathan V, Ramar N, Anto GJ, Meher SR. Evaluation of plan quality improvements in PlanIQ-guided Autoplanning. Rep Pract Oncol Radiother 2019;24(6):533-43. https://doi.org/10.1016/j. rpor.2019.08.003.

[15] Craft DL, Halabi TF, Shih HA, Bortfeld TR. Approximating convex Pareto surfaces in multiobjective radiotherapy planning: Approximating convex Pareto surfaces in multiobjective IMRT. Med Phys 2006;33(9):3399-407. https://doi.org/10.1118/ 1.2335486

[16] Craft D, McQuaid D, Wala J, Chen W, Salari E, Bortfeld T. Multicriteria VMAT optimization: Multicriteria VMAT optimization. Med Phys 2012;39(2):686-96. https://doi.org/10.1118/1.3675601.

[17] Kierkels RGJ, Visser R, Bijl HP, Langendijk JA, van 't Veld AA, Steenbakkers RJHM, Korevaar EW. Multicriteria optimization enables less experienced planners to efficiently produce high quality treatment plans in head and neck cancer radiotherapy. Radiat Oncol 2015;10(1). https://doi.org/10.1186/s13014-0150385-9. 